\title{
Experimental testing of self-healing ability of soft polymer materials
}

\author{
Nikolay V. Perepelkin (D) Jose M. Martin-Martinez • Alexander E. Kovalev • \\ Feodor M. Borodich $\cdot$ Stanislav N. Gorb
}

Received: 16 November 2018/Accepted: 25 February 2019/Published online: 14 March 2019

(C) The Author(s) 2019

\begin{abstract}
Bioinspired materials that act like living tissues and can repair internal damage by themselves, i.e. self-healing materials, are an active field of research. Here a methodology for experimental testing of self-healing ability of soft polymer materials is described. The methodology is applied to a recently synthesized polyurethane material Smartpol (ADHTECH Smart Polymers \& Adhesives S.L., Alicante, Spain). Series of tests showed that the material demonstrated self-healing ability. The tests included the following steps: each Smartpol specimen was cut in halves, then it was put together under compression, and after specified amount of time, it was pulled apart while monitoring the force in contact. The test conditions were intentionally chosen to be
\end{abstract}

N. V. Perepelkin · F. M. Borodich

School of Engineering, Cardiff University, Cardiff CF24 3AA, UK

N. V. Perepelkin $(\bowtie)$

Department of Applied Mathematics, National Technical

University "Kharkiv Polytechnic Institute", 2

Kyrpychova Str, Kharkiv 61002, Ukraine

e-mail: PerepelkinM@cardiff.ac.uk

J. M. Martin-Martinez

Adhesion and Adhesives Laboratory, University of

Alicante, 03080 Alicante, Spain

A. E. Kovalev · S. N. Gorb

Department of Functional Morphology and Biomechanics, Zoological Institute of the University of Kiel, Kiel,

Germany non-ideal. These non-idealities simultaneously included: (1) separation time was rather long (minutes and dozens of minutes), (2) there was misalignment of specimen parts when they were put together, (3) contacting surfaces were non-flat, and (4) repeated testing of the same specimens was performed and, therefore, repeated damage was simulated. Despite the above, the recovery of structural integrity (selfhealing) of the material was observed which demonstrated the remarkable features of Smartpol. Analysis of the experimental results showed clear correlation between adhesion forces (observed through the values of maximum pull-off force) and the time in contact which is a clear indicator of self-healing ability of material. It is argued that the factors contributing to self-healing of the tested material at macro-scale were high adhesion and strong viscoelasticity. The results of fitting the force relaxation data by means of mathematical model containing multiple exponential terms suggested that the material behaviour may be adequately described by the generalized Maxwell model.

Keywords Self-healing - Soft polymer ·

Polyurethane $\cdot$ Smartpol $\cdot$ Pull-off tests ·

Viscoelasticity 


\section{Introduction}

The idea of making a self-healing material has been attracting researchers for decades. Inspired by nature, the to-date advances in this area include wide variety of materials including polymers and polymeric composites [22, 28], nanocomposites [21], construction materials like concrete [24] and asphalt [20], and even metals [1]. Overall, self-healing materials have a great interest in a wide variety of applications in which once microcracks or cuts are produced they can be selfrepaired with time. This means that an ideal selfhealing material should recover the original material's performance in reasonable time [28].

The actual approaches to self-healing vary. Quite often self-healing materials are heterogeneous, this sort of materials deliver self-healing by means of included microscopic structures, such as capsules or microchannels [2, 28], shape memory wires, low melting point components [1], etc. However, encapsulation of healing agents in many cases results in the ability to perform self healing only once [21] which is a major drawback. In contrast, intrinsic self-healing materials do not have a dedicated microstructure containing healing agent but rather possess a latent self-healing functionality that is triggered by damage or by external agents [2].

Apart from homogeneity, another important characteristic of self-healing material is the ability to deliver intrinsic self-healing without the need of external agents (heat, pressure, radiation), i.e. the ability to be autonomous [21].

Polymers and polymer-based composites have huge variety of applications including such areas as civil engineering, transport, medicine, electronics etc. In the recent years, several self-healing polymeric materials have been developed and different mechanisms of self-healing have been proposed including the microencapsulation of the healing agent into the polymer formulation, the existence of reversible bonds, the creation of host-guest interactions, and the formation of non-covalent reversible bonds, among other [26]. More recently, different polyurethanes showing self-healing properties have been developed. For example, polyurethanes containing separate liquid isocyanate and polyol microcapsules have been designed [25] which, upon suffering a scratch or cut, can be repaired by chemical reaction between those encapsulated reactants [30]. However, the healed region was stiffer than the bulk and, therefore properties of the material were inhomogeneous and this may lead to stress concentration which, in turn, may significantly affect the performance of the healing material.

Using a very similar approach, two-component polyurethane clear coating based on isocyanate and polyol which heals small scratches under application of heat or sunlight has been proposed [18]. An alternative approach assumes that diamine molecule containing disulfide aromatic moiety is added during polyurethane synthesis for producing new urea groups upon crack formation, without the need of application of temperature or light irradiation to heal [19]. However, the results were given only for the selfhealing property developed after $1 \mathrm{~h}$. (It is worth noting that it is difficult to judge the effectiveness of self-healing processes when different studies are compared, as time-dependent aspects are often omitted in the literature). Similarly, polyurethanes containing diselenide bonds able to self-heal several times upon heat application have been proposed [6]. Furthermore, polyurethanes with self-healing property based on reversible reactions such as the Diels-Alder reaction activated by heat [3] or by UV radiation [16] have been proposed. Further studies have proposed polyurethanes that can self-heal via reversible bonding reactions [10]. Additionally, the synthesis of thermoplastic polyurethanes containing carbon nanotubes showing reversible self-healing that did not require sequestered healing agents has been proposed [9], and the microwave assisted self-healing thermoplastic polyurethane reinforced with graphene-carbon nanotubes has been recently proposed [13].

It is important to note that many of the above mentioned polyurethanes either require the intervention of external agents (pressure, radiation, heat) for self-healing to occur or the time needed for sufficient self-healing is too long. Recently, a new elastomeric polyurethane (Smartpol) with relatively fast selfhealing ability at room temperature and ability to recover large deformations has been synthesized at ADHTECH Smart Polymers \& Adhesives S.L. (Alicante, Spain). The preliminary tests at ADHTECH showed very promising results regarding the material ability to recover up to $83 \%$ of its structural strength after being damaged (see Sect. 2). However, more indepth study on ability of the material to self-heal under non-ideal conditions was needed. 
There exist various methods of assessment of selfhealing ability of materials. The review by $\mathrm{Wu}$, Meure et al. [28] suggests a number of approaches which vary from qualitative (visual inspection, puncture closure) to quantitative (testing of tensile strength, flexural strength, fracture toughness) methods. Self-healing of a hydrogel material was confirmed qualitatively in [15] by simply stretching the healed material by tweezers, and by the ability of the healed substance to hold its structure under its own weight. Optical observation of molecule exchange across the cut interface of hydrogel by means of fluorescent effects was used in [29]. In [5], optical observations were combined with rheological tests including experimental evaluation of storage and loss moduli of the tested material. In some works (e.g., [7, 26]), stress-strain curves of undamaged and healed material were analyzed. Also, Wool and O'Connor [27] in their paper categorized stages of self-healing in polymer materials and developed mathematical description of recovery processes.

In the present work, we studied the self-healing ability of the Smartpol material long time after manufacturing ( 9 months) under non-ideal recovery conditions. Namely, a series of pull-off tests was carried out in which specimens were cut in halves, put together for a specified amount of time and then pulled apart while continuously recording the applied force. During the tests, the cut specimens were put together after a dosens of minutes separation time interval. The cut surfaces were not perfectly flat and they were not ideally aligned during the tests.Also, repeated damage was simulated by using the same damaged specimen in repeated contacting and pulling apart. Despite all these factors that hindered self-healing, we were able to clearly observe increase in the pull-off force with respect to increasing contact time even in a short time range which is a good indication of self-healing. In our experiments, in less than 10 min time the material was able to restore its strength to the values comparable to the strength of undamaged material: $36 \%$ in the worst case, $68 \%$ in the best case.

The methodology used in the present study is similar to the work by Maes, Montarnal et al. [14] in which specimens made of supramolecular rubber were also put in contact and pulled apart while measuring the applied force. However, in that paper the initial damage of material was a brittle crack produced by means of specifically introduced inhomogeneities. To achieve a localized fracture, the specimens in [14] were fractured at low temperature (near the glass point of the rubber) and then heated back to the testing temperature. Hence, "clean and smooth surfaces are created without any noticeable deformation of the bulk" [14]. However, this kind of testing is somewhat far from real-life scenarios in which damage and healing of an autonomous material should occur at the same environmental conditions. In the present work, the maximum possible smoothness of the contacting interfaces was not required which also brings the present study closer to a real-life scenario. Also, in the present work, the same specimens were tested multiple times using pseudo-random test pattern of short, medium, and long contact duration times. This allowed us to confirm that indeed amount of adhesion force correlates with time in contact and does not depend on loading sequence.

The study shows that at macro-scale the factors contributing to self-healing of the Smartpol under nonideal conditions are high adhesion and strong viscoelasticity. Indeed, viscoelasticity allows stresses in the zone of contact to relax which results in an increase of the contact area (even if the contacting surfaces are non-smooth) and allows short-range inter-molecular interactions to take place. The results of fitting the force relaxation data by means of mathematical model containing multiple exponential terms suggest that the material behavior falls within the area of applicability of the generalized Maxwell model (the MaxwellWiechert model) [8].

It is known [11] that adhesion between polymer parts may have different physical origin (see also a comprehensive review by Myshkin and Kovalev [17]). In particular, it may be caused by van der Waals forces, the presence of surface charges, and different effects related to physi-/chemisorbtion. The observed adhesion of Smartpol was stronger than expected in standard polyurethanes in which hydrogen interactions between soft and hard segments are produced. Other polyurethanes showing self-healing contains reactive di-sulfide or di-tellurate groups that create new covalent bonds induced by heat or radiation. However, this is not the case of the Smartpol material. Hence, the nature of the actual adhesion mechanism in the material is the matter of further studies.

The rest of the paper is organized as follows. Section 2 contains information about the Smartpol material and some of its properties. In Sect. 3 the 
experimental set-up used for pull-off testing and the testing methodology are described. Experimental results of the repeated pull-off tests are presented in Sect. 4.

\section{The Smartpol material}

The Smartpol material [4] is a clear soft homogeneous substance (Figs. 1, 2) made of polyurethane with segmented molecular structure of hard and soft segments. In preliminary tests carried out at ADHTECH a metallic wire $(1 \mathrm{~mm}$ diameter) with 1 $\mathrm{kg}$ weight at its end was placed on the middle of rectangular section of a polyurethane specimen (Fig. 1). As the wire cut the polyurethane, the broken area restored its shape and structural integrity of the specimen at room temperature without external influence. To quantitatively characterize this ability another series of preliminary tests was carried out at ADHTECH using the Instron 4411 Universal testing machine (Instron Ltd., Buckinghamshire, UK) and dog-bone shaped specimens with the dimensions required by ISO 37-2 standard. The extent of selfhealing was tested by cutting the test sample by the middle and re-joining the two cut pieces immediately after cut in the same location of the cut, and left stand at $25^{\circ} \mathrm{C}$ for $24 \mathrm{~h}$. Afterwards, the tensile test was repeated and the mechanical properties were compared. The pulling rate was $50 \mathrm{~mm} / \mathrm{min}$. The mechanical properties of the Smartpol specimens before and after being cut are given in Table 1 . The results show

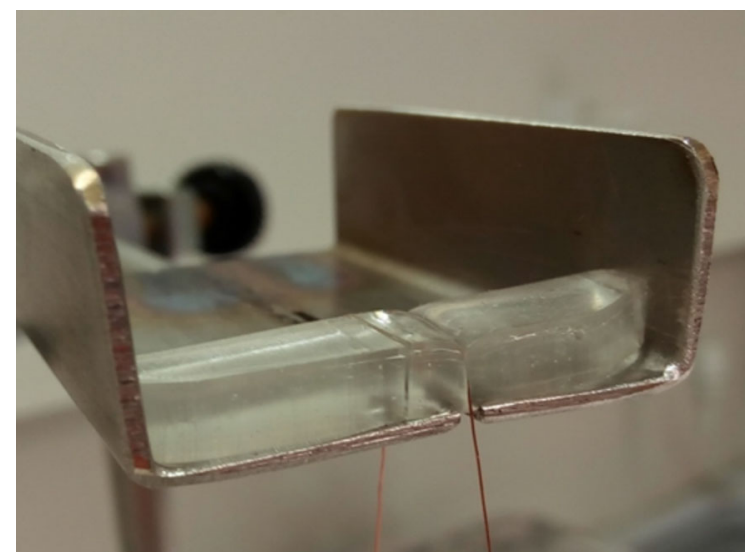

Fig. 1 The preliminary direct self-healing testing by means of cutting a specimen by a metallic wire

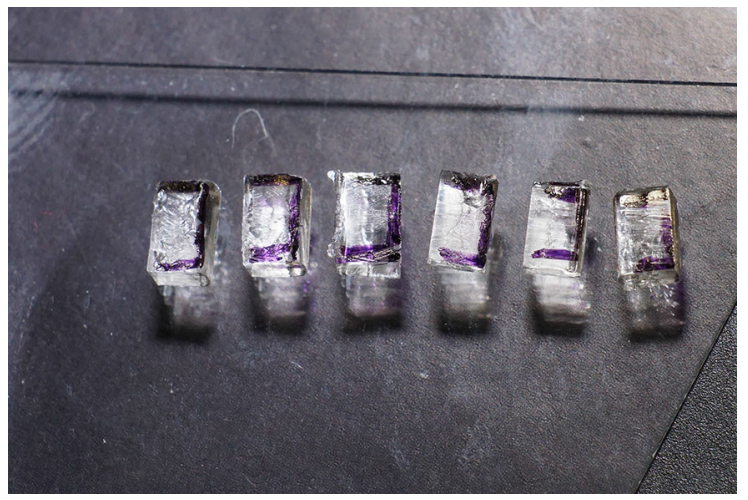

Fig. 2 The specimens used in pull-off tests

Table 1 Mechanical properties obtained from tensile testing of uncut and cut (after $24 \mathrm{~h}$ healing) Smartpol specimens

\begin{tabular}{lll}
\hline Property & Before cut & After cut \\
\hline Tensile strength (MPa) & 1.2 & 1.0 \\
Elongation-at-break (\%) & 873 & 932 \\
\hline
\end{tabular}

effective restoration of $83 \%$ of the mechanical strength of the material.

The subsequent sections of the present work discuss testing of Smartpol specimens under non-ideal conditions that simultaneously included long separation time, specimens misalignment and repeated damage.

\section{The experimental set-up for pull-off testing and the methodology}

In order to analyze the self-healing ability of the material under non-ideal conditions a number of pulloff tests was carried out. In the tests small prismatic specimens of nominal length $4 \mathrm{~mm}$ were used (Fig. 2). The cross-section dimensions of the specimens varied and were not specifically controlled. The tests were performed 9 months after manufacturing the sample.

During testing, each specimen was cut in half in the middle using a razorblade. The resulting halves were used in the experimental set-up as shown in Fig. 3. One half of each specimen was attached (glued) via an aluminium stub to the force sensor Futek (FUTEK Advanced Sensor Technology, Inc.). The opposite part of the sensor was attached to the rigid base (metal 


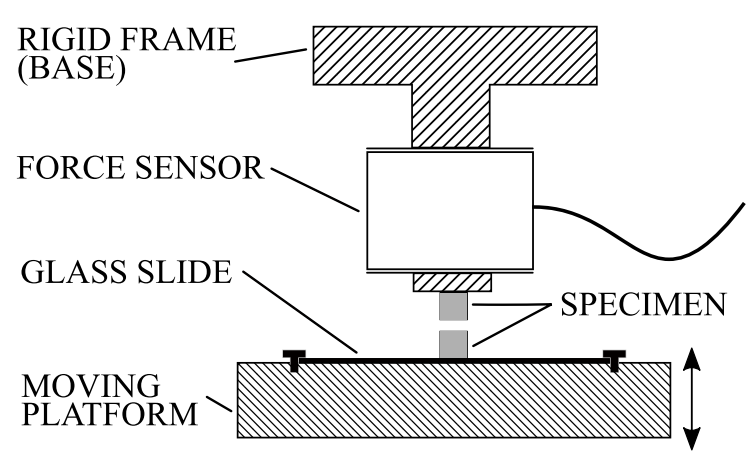

(a)

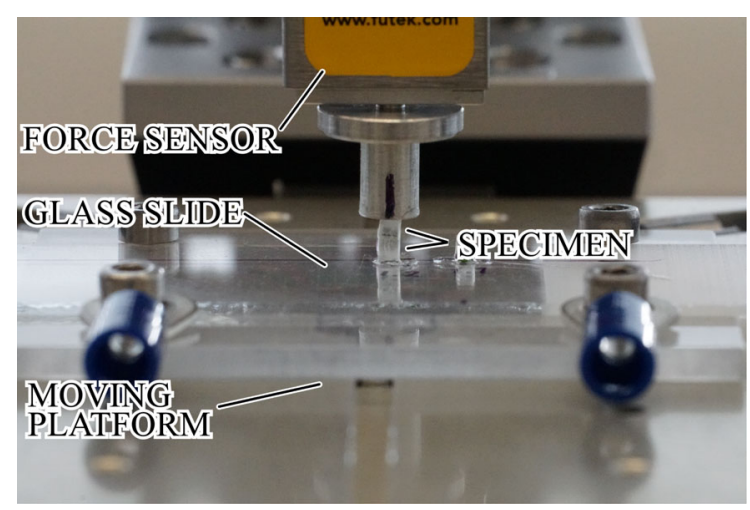

(b)

Fig. 3 The experimental set-up for direct testing of selfhealing: a schematic, b photographic image

frame). The other half of each specimen was glued (with Ergo 5925 Elastomer, Tagelswangen, Switzerland) to a glass slide. The latter was firmly attached to a moving metal platform. The platform movement was computer controlled by means of servo-drives through the Hexapod F-206S 6-axis motion controller [Physik Instrumente (PI) GmbH, Karlsruhe, Germany]. The hardware set-up was similar to the one used for adhesion studies in [12].

In the beginning of each test, the two halves of the specimen were aligned. After alignment, vertical displacement was assigned to the moving platform so that the two halves of the specimen were kept in compression. After having the specimen compressed for a specified amount of time (see test duration in Tables 2, 3), the moving platform returned to the initial position (the initial position was chosen to have the specimen halves fully separated after pulling-off). The approach and retraction speed was $3 \mathrm{~mm} / \mathrm{s}$. The tests were carried out at temperature $30^{\circ} \mathrm{C}$.
This kind of testing had multiple aims: (1) to confirm growth of adhesion between two pieces of the material with respect to time under non-ideal alignment conditions in a location where it had beed previously damaged (cut); (2) to study the ability of the material to withstand repeated damage in the same location; (3) to confirm the ability of the material to restore its structural integrity (self-healing) and to find out some of the contributing factors.

The force signal from the sensor was amplified, digitalized, and recorded using a MP100WSW data acquisition system and software AcqKnowledge 3.7.0 (Biopac Systems Inc., Goleta, CA, USA). As the readings demonstrated zero drift with respect to time, the experimental data for each individual test was corrected as follows. Let $P_{0}$ be the force reading at the time $t_{0}$ immediately before the test start and $P_{1}$ be the force reading immediately after the test end at the time $t_{1}$. The linear approximation was used for dependency of the zero drift with respect to time. Hence, the corrected value of force $P(t)$ at the time moment $t$ was obtained from the raw data $P_{r}(t)$ using the following formula:

$$
P(t)=P_{r}(t)-P_{0}-\left(P_{1}-P_{0}\right) \frac{t-t_{0}}{t_{1}-t_{0}}, \quad t \in\left[t_{0}, t_{1}\right] .
$$

Two series of pull-off tests were carried out during the experiment. In the former series of tests three specimens were tested using progressively increasing time in contact: 1, 2, 4, 8, 16 min. Due to limitations of assembly and alignment process, testing was not performed immediately after cutting the specimen in halves but rather after certain amount of time.

The purpose of the latter series of tests was to demonstrate that the amount of adhesion observed during separation tests correlates only with time in contact and does not depend on the loading sequence. To do so, another three specimens were manufactured and used in the same way as in the former test. However, the test pattern for each specimen was a pseudo-random combination of 6 tests with different duration: short (S), medium (M) and long (L) time in contact. Particular values of test durations are presented in Table 3.

As the material exhibited strong viscoelastic properties (see Sect. 4.3), each new test started 3 min after 
Table 2 The results of pull-off test series No. 1 (absolute values of force used)

\begin{tabular}{|c|c|c|c|c|c|c|}
\hline Specimen 1 & Test No. & 1 & 2 & 3 & 4 & 5 \\
\hline Time after cutting: $30 \mathrm{~min}$ & Test duration, $\min$ & 1 & 2 & 4 & 8 & 16 \\
\hline \multirow[t]{2}{*}{ Max. tensile strength: $0.70 \mathrm{MPa}$} & Maximum compressive force, $\mathrm{mN}$ & No data & 319.2 & No data & 297.2 & 279.7 \\
\hline & Maximum pull-off force, $\mathrm{mN}$ & 2235 & 2299 & 2404 & 2462 & 2581 \\
\hline Specimen 2 & Test No. & 1 & 2 & 3 & 4 & 5 \\
\hline Time after cutting: $12 \mathrm{~min}$ & Test duration, min & 1 & 2 & 4 & 8 & 16 \\
\hline \multirow[t]{2}{*}{ Max. tensile strength: $0.86 \mathrm{MPa}$} & Maximum compressive force, $\mathrm{mN}$ & 2043 & 1928 & 1798 & 1663 & 1519 \\
\hline & Maximum pull-off force, $\mathrm{mN}$ & 2934 & 3206 & 3314 & 3395 & 3455 \\
\hline Specimen 3 & Test No. & 1 & 2 & 3 & 4 & 5 \\
\hline Time after cutting: $16 \mathrm{~min}$ & Test duration, min & 1 & 2 & 4 & 8 & 16 \\
\hline \multirow[t]{2}{*}{ Max. tensile strength: $0.56 \mathrm{MPa}$} & Maximum compressive force, $\mathrm{mN}$ & 1217 & 1106 & 1005 & 937.7 & 796.6 \\
\hline & Maximum pull-off force, $\mathrm{mN}$ & 1791 & 1882 & 1967 & 2039 & 2139 \\
\hline
\end{tabular}

Table 3 The results of pull-off test series No. 2 (absolute values of force used)

\begin{tabular}{|c|c|c|c|c|c|c|c|}
\hline Specimen 1 & Test No. & 1 & 2 & 3 & 4 & 5 & 6 \\
\hline Time after cutting: $20 \mathrm{~min}$ & Test duration, min & 1 & 3 & 9 & 1 & 9 & 3 \\
\hline Test pattern: S/M/L/S/L/M & Maximum compressive force, $\mathrm{mN}$ & 281.3 & 246.6 & 211.5 & 197.9 & 184 & 145 \\
\hline Max. tensile strength: $0.43 \mathrm{MPa}$ & Maximum pull-off force, $\mathrm{mN}$ & 1220 & 1366 & 1511 & 1079 & 1354 & 1274 \\
\hline Specimen 2 & Test No. & 1 & 2 & 3 & 4 & 5 & 6 \\
\hline Time after cutting: $20 \mathrm{~min}$ & Test duration, min & 3 & 1 & 9 & 3 & 9 & 1 \\
\hline Test pattern: M/S/L/M/L/S & Maximum compressive force, $\mathrm{mN}$ & 961.6 & 832.8 & 828.2 & 710.9 & 700.4 & 647.2 \\
\hline Max. tensile strength: $0.81 \mathrm{MPa}$ & Maximum pull-off force, $\mathrm{mN}$ & 3084 & 2977 & 3318 & 3171 & 3302 & 3021 \\
\hline Specimen 3 & Test No. & 1 & 2 & 3 & 4 & 5 & 6 \\
\hline Time after cutting: $20 \mathrm{~min}$ & Test duration, min & 9 & 2 & 3.5 & 9 & 3 & 1 \\
\hline Test pattern: $\mathrm{L} / \mathrm{S} / \mathrm{M} / \mathrm{L} / \mathrm{M} / \mathrm{S}$ & Maximum compressive force, $\mathrm{mN}$ & 2339 & 1642 & 1638 & 1566 & 1379 & 1387 \\
\hline Max. tensile strength: $0.49 \mathrm{MPa}$ & Maximum pull-off force, $\mathrm{mN}$ & 2010 & 1735 & 1780 & 1901 & 1783 & 1678 \\
\hline
\end{tabular}

the previous one, in order to have residual stresses in specimens relaxed.

\section{The experimental results of pull-off tests}

\subsection{The analysis of the values of pull-off force}

The results of the pull-off tests with progressively increasing duration of contact are presented in Table 2. The results show gradual decrease in the absolute value of maximum compression force (most likely, due to incomplete relaxation of the specimens between tests). However, even despite this fact, clear correlation between the maximum recorded pull-off force and the test duration was observed. That is, adhesion between half-specimens increased as the time in contact grew. Graphically, dependency between the maximum pull-off force and the time in contact is represented in Fig. 4.

The results of the test series No. 2 are presented in Table 3. In graphical form these results are presented in Fig. 5. They clearly show that indeed the longer specimens stayed in contact the higher the pull-off force was regardless of the previous loading history. This kind of time-dependent adhesion demonstrate the ability of the material to repeatedly withstand destruction after being damaged and put together.

It should be noted that the presented data does not exhibit correlation between the amounts of compressive and pull-off forces. This can be explained by misalignment of the specimens and imperfections of 


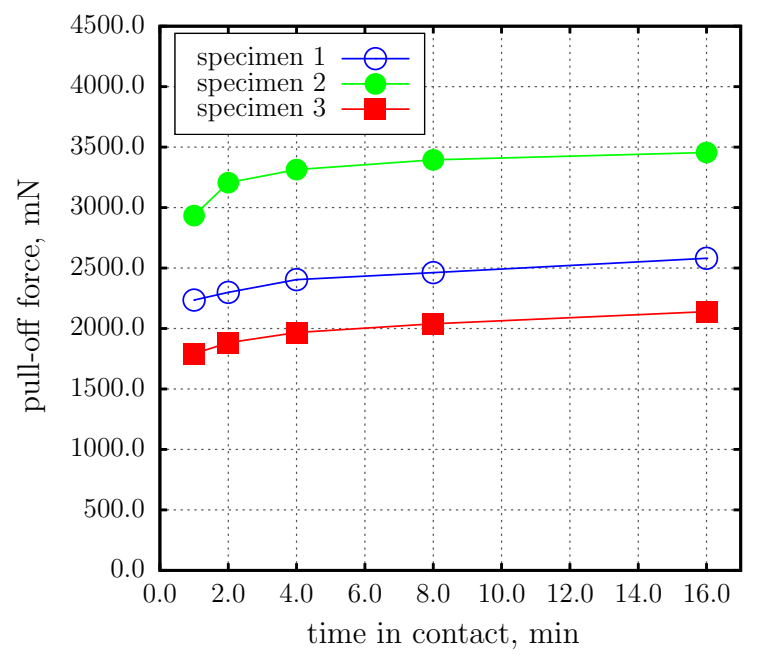

Fig. 4 Values of maximal pull-off force with respect to time in contact in the series of direct self-healing tests with progressively increasing duration of contact

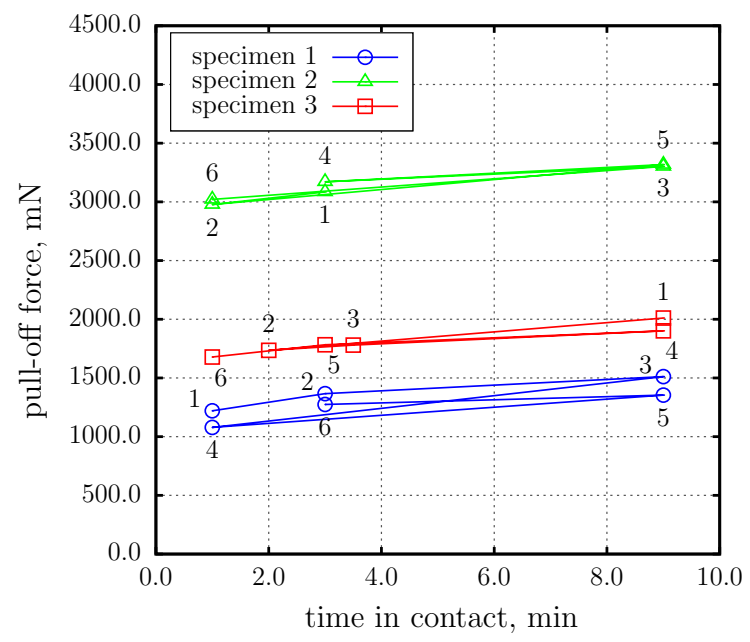

Fig. 5 Values of maximal pull-off force with respect to time in contact in the series of direct self-healing tests with pseudorandom load pattern. The numbers by the data points denote the test number in the corresponding sequence

contacting surfaces. Indeed, it was not possible to perfectly align the contacting surfaces to make the contact conditions optimal. Also, the contacting surfaces were not perfectly flat (Fig. 6). So, non-ideal contact conditions could reduce pull-off force drastically despite increased compressive force. In addition, it is likely that the areas of the established contact reached nearly the maximum possible values for each test, as the material was soft, sticky, and viscoelastic. However, it can be expected that the values of the

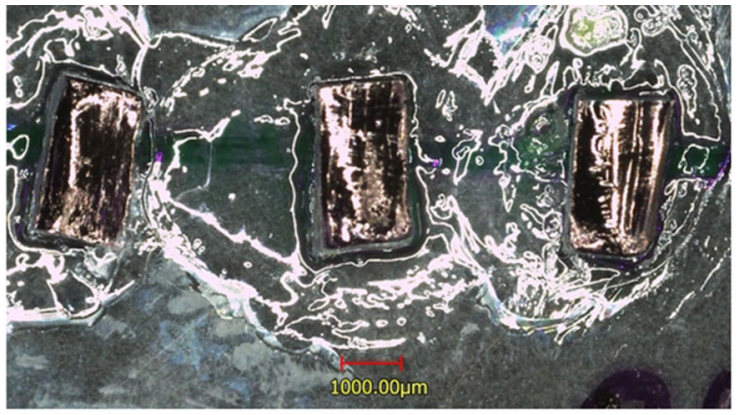

(a)

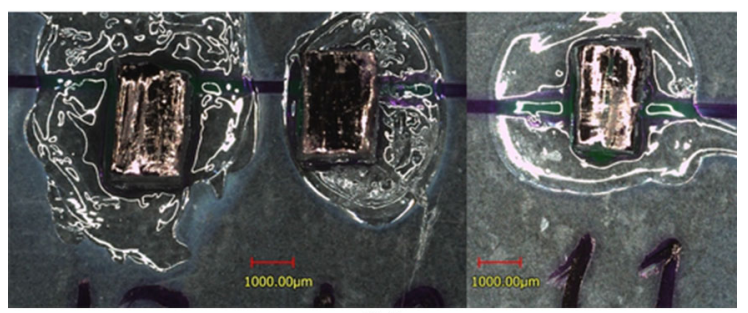

(b)

Fig. 6 The contacting surfaces (black) of the specimens used in the first (a) and the second (b) series of tests

compressive force may be important at very light loads.

The contacting areas of the specimens are depicted in Fig. 6. The images were obtained by means of the VR-3100 optical profilometer (KEYENCE Corp., Japan) in the observation mode after carrying out all the test series. To enhance the image contrast the contacting surfaces were painted black.

The cross section areas of the specimens were estimated to have the following values. Test series No.1: 3.7, 4.0, 3.8; test series No.2: 3.5, 4.1, 4.1 (in $\mathrm{mm}^{2}$, for the specimens 1,2 , and 3 , respectively). The mentioned cross section areas were just 'nominal' ones, i.e. the whole cross-sections. They were estimated using the ImageJ software using the images and corresponding length scales produced by the VR-3100 profilometer. The real contact areas were indeed different due to both misalignment and surface roughness.

Taking into account the maximum readouts of the tensile forces for each specimen (Tables 2, 3), the tensile strength of the cut specimens under non-ideal recovery conditions and high detachment speed was calculated to be $0.70,0.86,0.56 \mathrm{MPa}$ and $0.43,0.81$, $0.49 \mathrm{MPa}$ for specimens 1,2,3 in test series No.1 and 2, respectively. Thus, in less than 10 min time (test series 2) the material was able to restore its strength to the 
values comparable to the strength of undamaged material (Table 1): $36 \%$ in the worst case, $68 \%$ in the best case. However, due to non-ideal recovery conditions, the dispersion of the results was rather high.

High dispersion of the results show that individual imperfections and misalignment of specimens may influence the results much. Thus, it is advisable to include large number of specimens in self-healing tests with further statistical post-processing of the experimental data.

\subsection{Direct observations of detachment during pull-off tests}

It was observed that in order to fully separate the specimen halves, detachment had to be performed at high speeds and the target separation distance had to be rather long. Therefore, if the target separation distance was not long enough, the specimen halves did not separate fully and remain connected with a thin strip of material. That is, the two pieces of material started restoring its structural integrity and the actual self-healing occurred (Fig. 7). The detachment process during a pull-off test is depicted in Fig. 8.

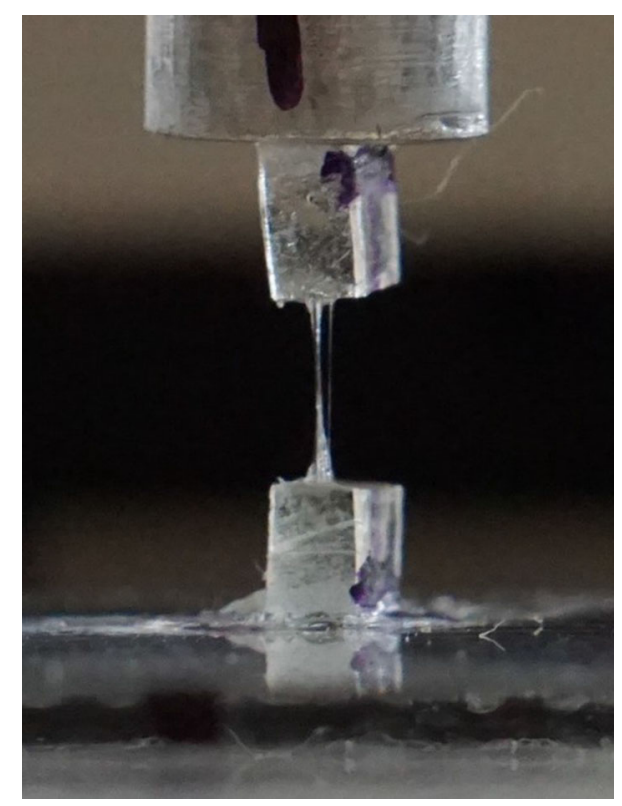

Fig. 7 Actual self-healing of a specimen observed when initial separation distance was chosen too short

\subsection{Analysis of viscoelastic behavior}

Viscoelasticity of the material was an important factor of its self-healing ability at macro-level. Clearly, to achieve effective self-healing under non-ideal conditions, e.g. surface roughness of contacting surfaces, it is important to: (1) maximize the contact area, (2) make the healed zone have the same physical properties as the undamaged homogeneous material whenever possible. Without viscoelasticity, the real contact area during autonomous healing is determined by the energy equilibrium of contacting asperities that keeps the gaps between surfaces of the solid parts. In addition, absence of full contact creates inhomogeneity of the stress field resulting in high variability of the field. The effect of the viscoelastic behaviour of the material is twofold: (1) it reduces the stress field inhomogeneities; and (2) the strain energy accumulated in the asperities decreases and the true contact area increases, that, in turn, increases the adhesive interactions between contacting surfaces.

In the present subsection some qualitative data gathered during the pull-off tests is presented.

The observed viscoelastic behavior of the material was very strong. Fig. 9a demonstrates large deformations of a strip specimen after being gripped in a mechanical grip for just $15 \mathrm{~min}$. Remarkably, in $14 \mathrm{~h}$. the specimen had mostly restored its initial shape (Fig. 9b).

During the pull-off tests the values of longitudinal force acting on the specimens were continuously recorded. The data confirmed strong viscoelastic behavior of the material. As an example, typical readings during the test with progressively increasing duration are presented in Fig. 10. All the curves correspond to the same specimen. Note that the figure presents the corrected data according to the formula (1). Negative values of force correspond to compression, positive ones correspond to tension.

It was found that force-time readings could be well fit with an exponentially decaying function. The fitting was performed as follows. Let $P_{c m p}$ be the maximum absolute value of compressive force during the test and $t_{c m p}$ the corresponding moment of time. The fitting was applied to the data points between time $t_{c m p}$ and the initial moment of pulling off. To equally treat different data sets with different time reference points and different force magnitudes, the following change of 


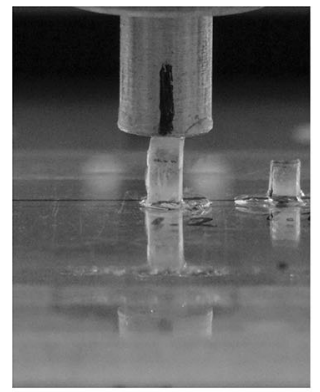

(a)

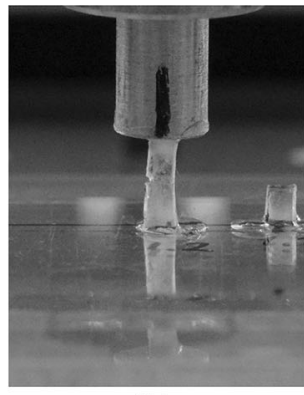

(b)

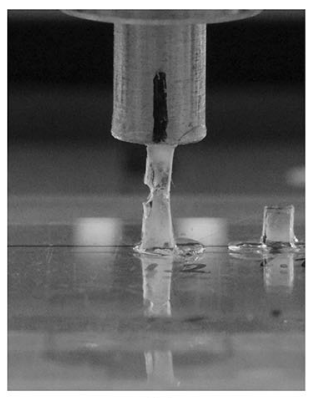

(c)

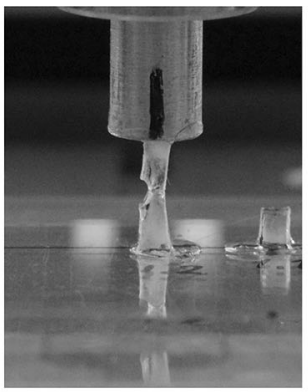

(d)

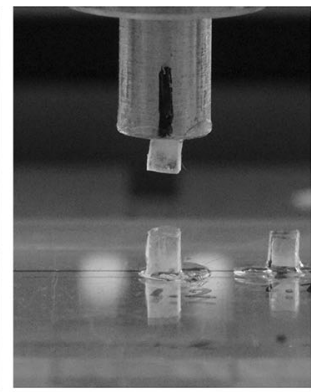

(e)

Fig. 8 The sequence of photographs illustrating detachment progress a-e of the two specimen halves during the pull-off test. a The test start, $\mathbf{e}$ the test end

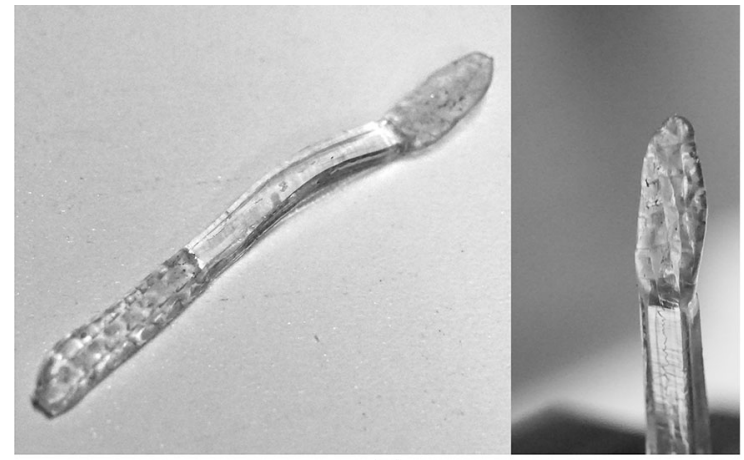

(a)

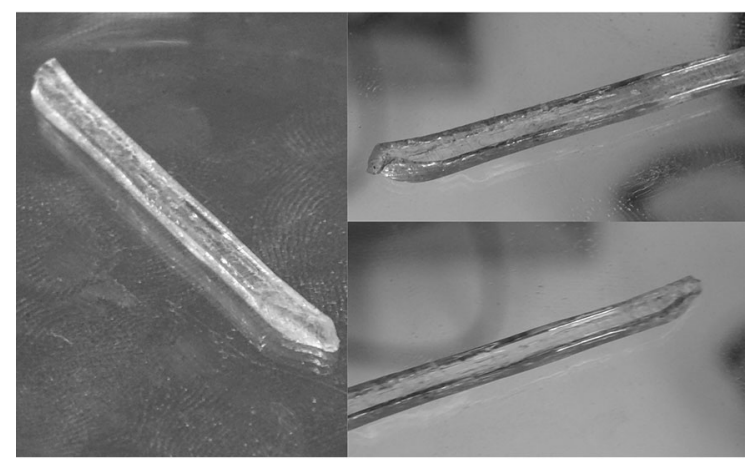

(b)

Fig. 9 a Large deformations of the material strip after being gripped for $15 \mathrm{~min}$ by a mechanical grip. b After $14 \mathrm{~h}$ the shape had mostly been restored

variables was done: $\tau=t-t_{c m p}, \hat{P}=P / P_{c m p}$. The modified data was fitted by means of the least-squares curve fitting to the following approximation:

$\hat{P}_{f i t}=-1+\sum_{i=1}^{M} a_{i}\left(1-\exp \left(-\lambda_{i} \tau\right)\right)$
In Fig. 11 the results of fitting a 9 min. data set (test series No. 2, specimen No. 1, test No. 3) are presented. The number $M$ of exponential terms in (2) varies from 1 to 4 . Graph comparison shows that at least 3 or 4 term approximation is needed to effectively fit the experimental data for both short and long time scales simultaneously.

Small number of used exponential terms show that the material behavior can be well described by the generalized Maxwell viscoelastic model (the Maxwell-Wiechert model) [8].

Additionally, to study how approximation coefficients vary between specimens the fitting coefficients for all the 9 min test in the test series No. 2 were computed using 4-term approximation. The results are shown in Table 4. The results show that the fast decaying terms tend to vary between specimens while slowly decaying ones remain almost the same for all specimens. As fast decaying terms dominate during the initial loading stage, these variations can be explained by difference in the individual features of the geometry of the specimens and individual misalignment. These individual differences between the specimens influence the overall system behavior while contact is not fully established.

Remark The author of [23] used an approximation of the same structure as (2) to construct Prony series for the elastic modulus of a material. It is argued in [23] that fitting experimental data with an approximation of type (2) may require a weight function as at long test duration most data is dominated by slowly decaying exponents. However, the matter of construction of the best approximation is out of scope of the present paper and is not discussed here. 


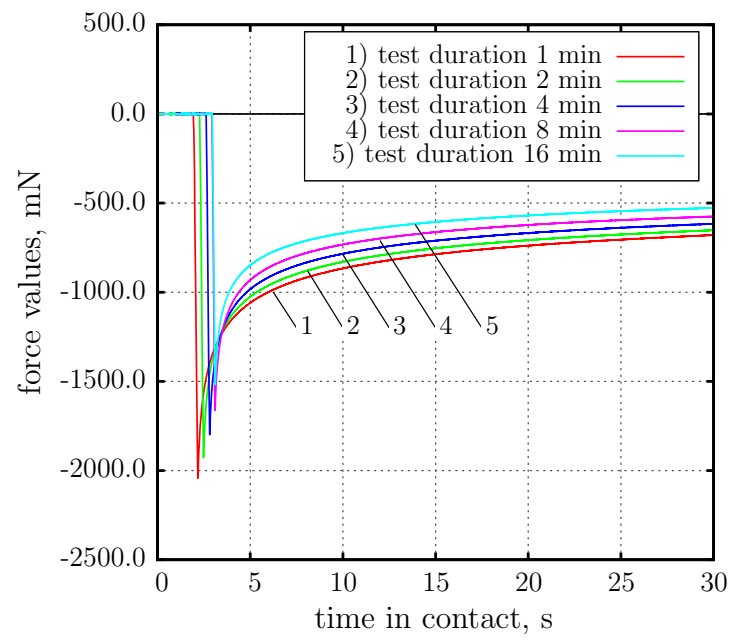

(a)

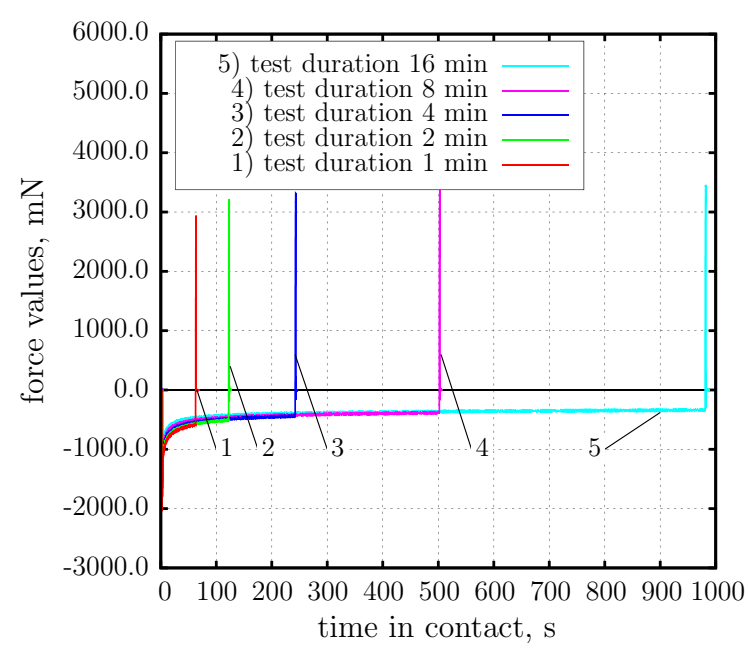

(b)

Fig. 10 Typical force readings corresponding to the same specimen during the pull-off test with progressively increasing test duration. a The initial stage. b The complete force-time curves

\section{Conclusions}

In the present work a methodology for experimental testing of self-healing ability of soft polymer materials is described. The methodology is applied to a recently synthesized polyurethane material Smartpol (developed at ADHTECH Smart Polymers \& Adhesives S.L., Alicante, Spain). The purpose of the tests was to show that the material demonstrates self-healing after being damaged (cut) under non-ideal conditions. The non-ideality of the test conditions simultaneously

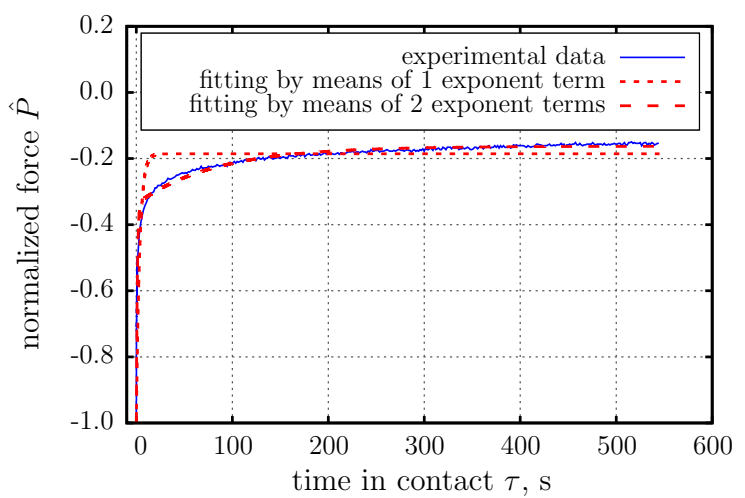

(a)

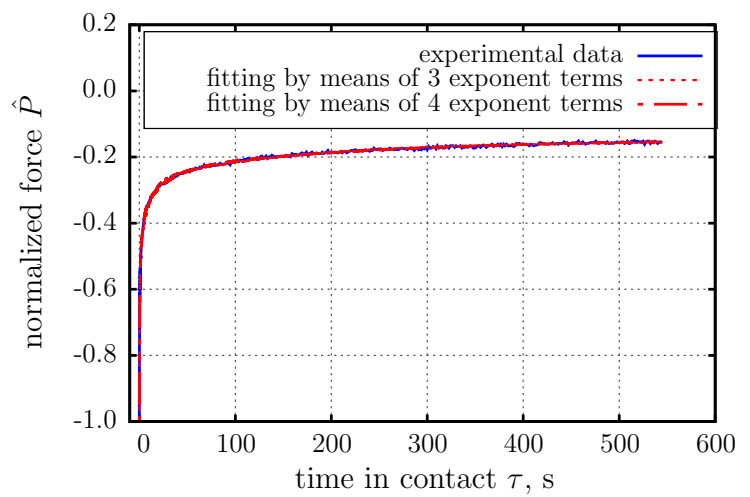

(b)

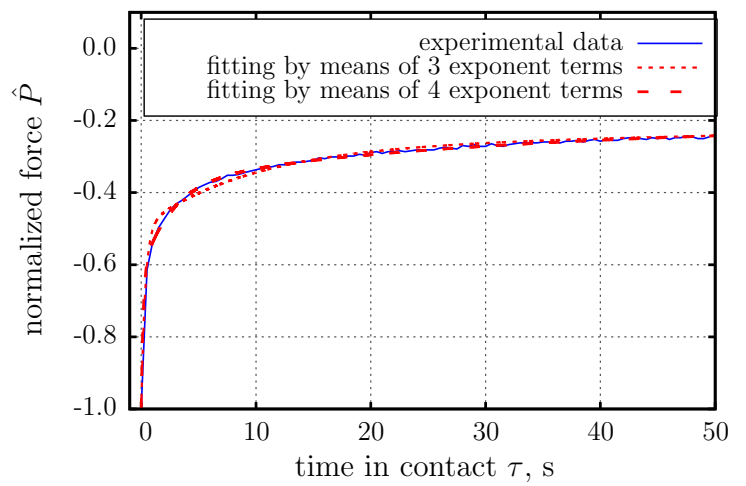

(c)

Fig. 11 Example results of fitting experimental data using different number of exponent terms. a 1 and 2 terms used; b 3 and 4 exponent terms used, 3 and 4 exponent terms used, initial loading stage is shown (the density of shown experimental data points was reduced to improve image clarity)

included: (1) long separation time (minutes and dozens of minutes), (2) misalignment of specimens when put together, (3) non-flat contacting surfaces, (4) repeated testing of the same specimens which simulated repeated damage before full self-healing 
Table 4 The fitting coefficients for 9 min tests of the series No. 2

\begin{tabular}{lllllllll}
\hline Fitting coefficients & $a_{1}$ & $a_{2}$ & $a_{3}$ & $a_{4}$ & $\lambda_{1}$ & $\lambda_{2}$ & $\lambda_{3}$ & $\lambda_{4}$ \\
\hline Specimen 1 & & & & & & & & \\
$\quad$ Run 1 & 0.379 & 0.242 & 0.131 & 0.102 & 6.08 & 0.387 & 0.0423 & 0.00443 \\
$\quad$ Run 2 & 0.370 & 0.240 & 0.135 & 0.103 & 6.50 & 0.428 & 0.0457 & 0.00452 \\
Specimen 2 & & & & & & & & \\
$\quad$ Run 1 & 0.336 & 0.230 & 0.134 & 0.104 & 4.75 & 0.353 & 0.0417 & 0.00443 \\
Run 2 & 0.331 & 0.228 & 0.134 & 0.0905 & 5.10 & 0.369 & 0.0433 & 0.00486 \\
Specimen 3 & & & & & & & & \\
$\quad$ Run 1 & 0.319 & 0.231 & 0.142 & 0.119 & 4.39 & 0.334 & 0.0397 & 0.00450 \\
Run 2 & 0.305 & 0.217 & 0.131 & 0.0953 & 4.26 & 0.315 & 0.0382 & 0.00419 \\
\hline
\end{tabular}

occurred. During the tests, the specimens were cut in halves, then put together with compression and pulled apart after specified amount of time while monitoring the applied force.

Overall, the material demonstrated remarkable properties: (1) strong time-dependent adhesion (in fact, in less than 10 min time the material was able to restore its strength to the values comparable to the strength of undamaged material (Table 1): $36 \%$ in the worst case, $68 \%$ in the best case); (2) strong viscoelasticity (during compression, the amount of compressive force reduced by $50 \%$ of the initial value in a few seconds time); (3) ability to recover large deformations of dozens of percent (Fig. 9).

The actual self-healing was observed during pulloff tests (Fig. 7). If the target separation distance was not long enough, the specimen halves did not separate fully as the two pieces of material started restoring its structural integrity during compression phase of the tests.

Multiple repeated pull-off tests demonstrated that the longer amount of time specimens stayed in contact the higher the pull-off force was regardless of the previous loading history (to confirm this test durations were chosen in a pseudo-random manner). This kind of time-dependent adhesion demonstrated the ability of the material to repeatedly withstand destruction after being damaged and put together. Increase of adhesion with respect to time in contact (observed through the values of maximum pull-off force) was also a clear precursor of self-healing ability of material.

The study showed that at macro-scale the factors contributing to self-healing of the tested material were high adhesion and strong viscoelasticity. Indeed, viscoelasticity allows stresses in the zone of contact to relax which increases contact area (even if the contacting surfaces are non-smooth) and allows shortrange inter-molecular interactions to take place. The results of fitting the force relaxation data by means of mathematical model containing multiple exponential terms suggested that the material behavior may be well described by the generalized Maxwell model (the Maxwell-Wiechert model) [8].

Acknowledgements Authors express their gratitude to the European Network of Bioadhesion Expertise: Fundamental Knowledge to Inspire Advanced Bonding Technologies, COST Action CA15216.

Funding Dr. Nikolay Perepelkin acknowledges that his participation in this project has been funded from the European Union's Horizon 2020 research and innovation programme under the Marie Sklodowska-Curie Grant Agreement No. 663830. Prof. Feodor Borodich acknowledges that his visit the Functional Morphology and Biomechanics Group at Kiel University (October-December 2017) was funded by the Alexander von Humboldt Foundation within Renewed Research Stays Programme.

\section{Compliance with ethical standards}

Conflict of interest Dr. Nikolay Perepelkin acknowledges his double affiliation to Cardiff University, Cardiff, UK and National Technical University "Kharkiv Polytechnic Institute", Kharkiv, Ukraine.

Open Access This article is distributed under the terms of the Creative Commons Attribution 4.0 International License (http:// creativecommons.org/licenses/by/4.0/), which permits unrestricted use, distribution, and reproduction in any medium, provided you give appropriate credit to the original author(s) and the source, provide a link to the Creative Commons license, and indicate if changes were made. 


\section{References}

1. Alaneme KK, Bodunrin MO (2017) Self-healing using metallic material systems-a review. Appl Mater Today 6:9-15

2. Blaiszik BJ, Kramer SLB, Olugebefola SC, Moore JS, Sottos NR, White S (2010) Self-healing polymers and composites. Ann Rev Mater Res 40:179-211

3. Chen X, Dam MA, Ono K, Mal A, Shen HB, Nutt SR, Sheran K, Wudl F (2002) A thermally re-mendable crosslinked polymeric material. Science 295:1698-1702

4. Colera Llavata M, Costa Vayá V, Jofre Reche JA, Martín Martínez JM (2016) Self-healing polyurethane polymers. Patent No. EP 3103846 A1, Spain

5. Ding F, Wu S, Wang S, Xiong Y, Li Y, Li B, Deng H, Du Y, Xiao L, Shi X (2015) A dynamic and self-crosslinked polysaccharide hydrogel with autonomous self-healing ability. Soft Matter 11(20):3971-3976

6. Du W, Jin Y, Pan J, Fan W, Lai S, Sun X (2018) Thermal induced shape-memory and self-healing of segmented polyurethane containing diselenide bonds. J Appl Polym Sci 135(22):46326

7. Gulyuz U, Okay O (2013) Self-healing polyacrylic acid hydrogels. Soft Matter 9(43):10287-10293

8. Gutierrez-Lemini D (2013) Engineering viscoelasticity. Springer, New York

9. Harmon JP, Bass R (2014) Self-healing polycarbonate containing polyurethane nanotube composite. Patent No. 8846801, USA

10. Higaki Y, Otsuka H, Takahara A (2006) A thermodynamic polymer cross-linking system based on radically exchangeable covalent bonds. Macromolecules 39:2121-2125

11. Kovalev A, Sturm H (2013) Polymer adhesion. In: Wang QJ, Chung Y-W (eds) Encyclopedia of tribology. Springer, Berlin, pp 2551-2556

12. Kovalev AE, Gorb SN (2012) Charge contribution to the adhesion performance of polymeric microstructures. Tribol Lett 48:103-109

13. Li Y, Gao F, Xue Z, Luan Y, Yan X, Guo Z, Wang Z (2018) Synergistic effect of different graphene-cnt heterostructures on mechanical and self-healing properties of thermoplastic polyurethane composites. Mater Des 137:438-445

14. Maes F, Montarnal D, Cantournet S, Tournilhac F, Corte L, Tournilhac F, Corté L, Leibler L (2012) Activation and deactivation of self-healing in supramolecular rubbers. Soft Matter 8(5):1681-1687

15. Mukherjee S, Hill MR, Sumerlin BS (2015) Self-healing hydrogels containing reversible oxime crosslinks. Soft Matter 11(30):6152-6161
16. Muscas F, Navarro R, Seoane-Rivero R, Cuevas JM, Ureña A, Marcos-Fernández A (2018) Self-healing behaviour in polycaprolactone-based polyurethanes. In: UTECH Europe 2018

17. Myshkin N, Kovalev A (2018) Adhesion and surface forces in polymer tribology-a review. Friction 6:143-155

18. Rad AS (2007) Automotive clear coat polyurethane (desmodur z4470+ different desmophen) with respect to some physical and self-healing properties. Trends Appl Sci 7(5):400-406

19. Rekondo A, Martín R, de Luzuriaga AR, Cabañero G, Grande HJ, Odriozola I (2014) Catalyst-free temperature self-healing elastomers based on aromatic disulfide metathesis. Mater Horizons 1:237-240

20. Sun D, Sun G, Zhu X, Guarin A, Li B, Dai Z, Ling J (2018) A comprehensive review on self-healing of asphalt materials: mechanism, model, characterization and enhancement. Adv Colloid Interface Sci 256:65-93

21. Thakur VK, Kessler MR (2015) Self-healing polymer nanocomposite materials: a review. Polymer 69:369-383

22. Trask RS, Williams HR, Bond IP (2007) Self-healing polymer composites: mimicking nature to enhance performance. Bioinspir Biomim 2(1):1-9

23. Tzikang C (2000) Determining a Prony series for a viscoelastic material from time varying strain data. NASA Langley Technical Report Server. https://ntrs.nasa.gov/ archive/nasa/casi.ntrs.nasa.gov/20000052499.pdf

24. van Tittelboom K, de Belie N (2013) Self-healing in cementitious materials-a review. Materials 6:2182-2217

25. Wang W, Xu L, Li X, Lin Z, Yang Y, An E (2014) Selfhealing mechanisms of water triggered smart coating in seawater. J Mater Chem A 2:1914-1921

26. Wool RP (2008) Self-healing materials: a review. Soft Matter 4:400-418

27. Wool RP, O'Connor KM (1998) A theory crack healing in polymers. J Appl Phys 52:5953-5963

28. Wu DY, Meure S, Solomon D (2008) Self-healing polymeric materials: a review of recent developments. Prog Polym Sci 33:479-522

29. Xu Z, Peng J, Yan N, Yu H, Zhang S, Liu K, Fang Y (2013) Simple design but marvelous performances: molecular gels of superior strength and self-healing properties. Soft Matter 9(4):1091-1099

30. Yang J, Keller MW, Moore JS, White SR, Sottos NR (2008) Microencapsulation of isocyanates for self-healing polymers. Macromolecules 41(24):9650-9655

Publisher's Note Springer Nature remains neutral with regard to jurisdictional claims in published maps and institutional affiliations. 